\title{
Dorsal skinfold titanium chamber for non-invasive imaging in nude mice using multiphoton and harmonic generation microscopy
}

\author{
Feng-Chieh $\mathrm{Li}^{1}$, Chun-Chin Wang ${ }^{1}$, Sung-Jan Lin ${ }^{2}$, Shiou-Hwa Jee ${ }^{2}$, Chen-Yuan Dong ${ }^{1 *}$ \\ 1: Microscopic Biophysics Laboratory, Department of Physics, National Taiwan University, \\ Taipei, Taiwan 106, ROC \\ 2: Department of Dermatology, National Taiwan University Hospital and College of \\ Medicine, Taipei, Taiwa 100, ROC
}

*To whom correspondence should be addressed : cydong@phys.ntu.edu.tw

Tel.: 886-2-3366-5155 ; Fax : 886-2-3366-5244

\begin{abstract}
For many years, the dorsal skinfold chamber has been used for intravital observation in animal models. We have developed a chamber built using medical-grade titanium. Our design allows long-term, high resolution, and large-area observation in nude mice in vivo. Combined with two-photon fluorescence and second-harmonic generation microscopy, our chamber can be used to obtain qualitative and quantitative information for in vivo analysis of diseases such as tumors.
\end{abstract}

\section{INTRODUCTIONS}

Many areas of biomedical research rely on in vitro observation of single-cell behavior. However, issues in biomedical research are best addressed using in vivo research methods as they best simulate the behaviors of biological systems of interest. Therefore, the ability to obtain qualitative and quantitative information from the biological systems using intravital techniques remains a critical of life science research.

One aspect of intravital research involves the design of mechanical devices allowing in-vivo observation of biological phenomena. One such example is the dorsal skinfold chamber. ${ }^{[1][2][3]}$ This presentation describes the design of a titanium (medical-grade) skinfold chamber which can be used for in vivo microscopic observations. Our design is unique in that it allows high resolution imaging using high numerical aperture objectives. This design may be applied to reveal mechanistic pathways of important physiological processes such as tumor metastasis.

\section{METHODS AND MATERIALS}

\section{Chamber Design}

Our version of the chamber design involves an observation window using a No. $1.5,15 \mathrm{~mm}$ circular cover glass. A cylinder-shape push piece located on the opposite side of chamber is used to adhere the tissue to the observation cover glass. To be compatible with biological samples, this chamber is made of medical-graded titanium alloy (6Al-4V, ELI). To allow repeated imaging of the same area within the specimen, three target marks were made on the chamber for position determination. Our design is shown in Fig. 1.

\section{Animal Model}

Our design is intended to be used on 8-week-old nude mice (BALB/c-nu, National Animal Center, Taiwan). Those mice were processed in a laminar flow. For feasibility studies, GFP human melanoma tumor cell line were implanted in the mouse for long-term observation.

Experiment Setup

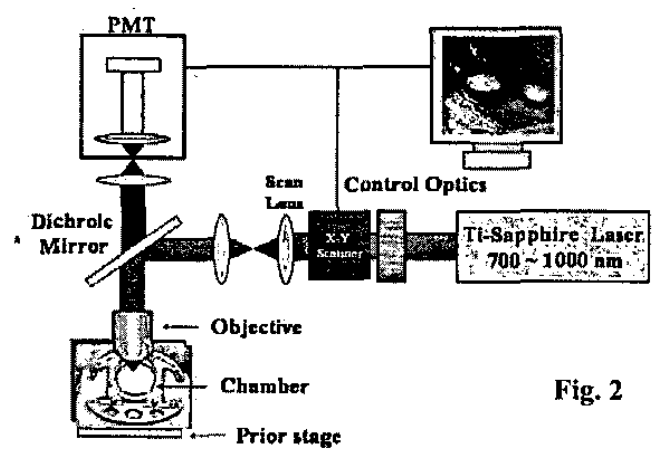




\section{THP-29(T5)}

We choose to use two-photon fluorescence and second-harmonic generation microscopy as imaging techniques. Our microscope is home-built from an upright microscope (E800, Nikon, Japan) and the multiphoton laser wavelength used was $780 \mathrm{~nm}$. A schematic of the two-photon microscope used in this study is show in Fig. 2.

\section{RESULTS}

GFP-expressing tumor cells are injected into the chamber-sealed skin for incubation and observation. With the applications of two-photon ${ }^{[4]}$ and second harmonic generation microscopy (minimally-invasive, non-linear, microscopic techniques), the GFP labeled tumor mass (green) and rhodamine dextran filled blood vessels (red) were visible through the imaging window (Figs. 3 and 4). The large-area imaging of collagen and blood vessels is shown in Fig. 5
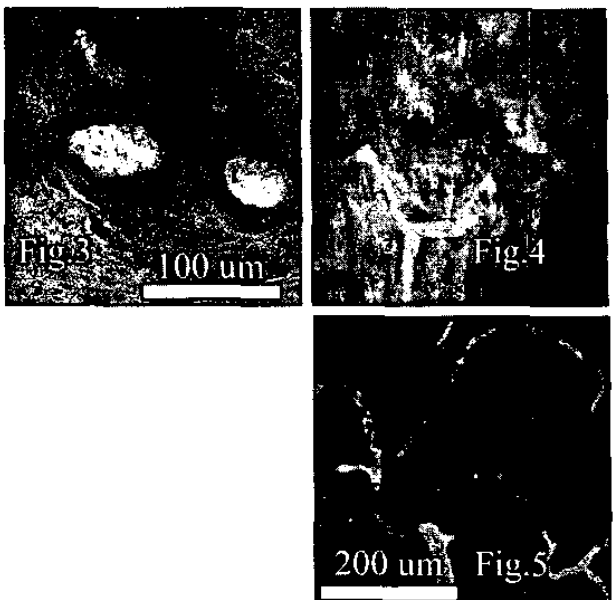

\section{CONCLUSIONS}

We have demonstrated our design of the biocompatible dorsal skinfold chamber can be used to obtain images of tumor physiology in vivo. Combined with two-photon imaging techniques, we plan to use the chamber to investigate tumor growth phenomena, metastasis, and mechanisms of angiogenesis in vivo. This method also may be applicable to studying other physiological processes in vivo.

\section{REFERENCES}

[1] Q. Huang, et al. "Noninvasive visualization of tumors in rodent dorsal skin window chambers." Nature Biotechnology, 17, pp. 1033-1035(1999).

[2] E.B. Brown, R.B. Campbell, Y. Tsuzuki, et al. "In vivo measurement of gene expression, angiogenesis and physiological function in tumors using multiphoton laser scanning microscopy "Nature Medicine, 7, pp. 864-868(2001).

[3] H.A. Lehr, M. Leunig, M.D. Menger, et al. "Dorsal skinfold chamber technique for intravital microscopy in nude mice." American Journal of Pathology, 143, pp. 1055-1062(1993).

[4] P.So, C.Y. Dong, B.R. Masters, et al. "Two-photon excitation fluorescence microscopy. " Annual Review of BiomedicalEengineering, 2,pp. 399-429(2000). 